\title{
La formación universitaria en periodismo. ¿Mera capacitación tecnológica o una formación integral en el nuevo ecosistema informativo?
}

\author{
José Vicente GARCÍA-SANTAMARÍA \\ Universidad Carlos III de Madrid \\ jvicente.santamaria@telefonica.net \\ Alejandro BARranquero CARretero \\ Universidad Carlos III de Madrid \\ abarranq@hum.uc3m.es
}

\begin{abstract}
Resumen:
Este artículo propone un acercamiento a algunos de los principales retos a los que hoy se enfrenta la formación universitaria en periodismo frente a un escenario laboral marcado por los vaivenes constantes de la digitalización. El estudio se apoya en una revisión de la literatura académica de referencia, así como en los resultados de una investigación cualitativa acerca de nuevos modelos de negocio en prensa digital, de los que se infiere un retrato del perfil profesional demandado por los diarios online.
\end{abstract}

Palabras clave: Formación en periodismo; digitalización; convergencia; periodismo digital.

\section{University journalism education. Mere technological training or comprehensive education in the new information environment?}

\begin{abstract}
:
This paper approaches the main challenges of university journalism education facing a new labour market defined by the permanent changes brought about by digitalization. The methodology is based both on an extensive review of referential academic literature and on the first results of a qualitative research on new business models in the digital press, from which we define the most demanded professional profile.
\end{abstract}

Key Words: journalism education; digitalization; convergence; online journalism.

\section{Referencia normalizada:}

García Santamaría, J.V. y Barranquero Carretero, A. (2014): La formación universitaria en periodismo. ¿Mera capacitación tecnológica o una formación integral en el nuevo ecosistema informativo?. Historia y Comunicación Social. Vol. 19. Núm. Especial Enero. Págs. 641-651.

Sumario: 1. Presentación del estudio. 2. La formación periodística en el entorno digital. 3. Algunas pistas para abordar el futuro. 4. Conclusiones. 5. Bibliografía. 


\section{Presentación del estudio}

Las siguientes líneas plantean un acercamiento a los principales desafíos a los que hoy se enfrenta la enseñanza del periodismo en un escenario digital que, entre otras mutaciones, ha acabado por modificar los antiguos estándares de trabajo en las redacciones ${ }^{1}$. Partimos de la hipótesis de que las técnicas y soportes informativos evolucionan con tanta rapidez que las facultades de periodismo de todo el mundo deben realizar una revisión constante de sus enseñanzas, con el objeto de evitar que se produzcan desfases entre la formación recibida en las aulas y las demandas de un entorno laboral en constante transformación.

En la senda de clásicos en la materia como Mark Deuze (2008: 350), consideramos que no existe una única vía para reflexionar acerca de la formación en el nuevo ecosistema digital puesto que los problemas que se dirimen hoy no son necesariamente nuevos, pero tampoco exactamente antiguos, y, de hecho, los propios debates contemporáneos se asemejan a los que ya se suscitaban hace más de dos décadas (Stepbenson, 2011: 12).

La metodología se apoya en una investigación acerca de nuevos modelos de negocio en prensa digital, a partir de un estudio Delphi en el que han participado editores y directores de 25 medios de comunicación. Dentro de este, nos apoyamos en las respuestas de los informantes-clave acerca de la organización de las nuevas redacciones y los procesos de formación y selección de personal.

En segundo lugar, nos interesa contrastar la opinión suscitada en el propio campo profesional con los principales debates que hoy emergen en universidades de referencia, y su eco en las principales revistas académicas que han abordado esta materia. El background anterior se completa con una revisión de informes profesionales acerca del perfil del nuevo periodista, difundidos por centros de avalado prestigio como la Asociación de la Prensa de Madrid (2012), el Reuters Institute for the Study of Journalism (2012) o el Pew Research Center's Project for Excellence in Journalism (2012, 2013), en particular The State of the News Media.

\section{La formación periodística en el entorno digital}

Los debates acerca de cuáles son los conocimientos y destrezas más adecuados para la capacitación de los futuros profesionales del periodismo han sido objeto de una persistente polémica en las últimas décadas, hasta el punto de ser calificados de "esquizofrénicos" (Sloan, 1990: 4) debido a su extrema diversidad y a los constantes desencuentros entre academia y entorno profesional.

Estas discusiones se han venido suscitando, al menos, desde las primeras iniciativas educativas surgidas a mediados del siglo XIX en el contexto de la industrialización estadounidense. Sin embargo, los primeros análisis empíricos acerca del perfil profesional del periodista datan de finales de la Segunda Guerra Mundial, y comien- 
zan a incrementarse sustancialmente a partir de la década de los setenta del siglo pasado, cuando se insiste en retratar la profesión a fin de adecuar la enseñanza a las reorganizaciones constantes del newsroom que conlleva cada nuevo soporte.

Ya desde finales de siglo XX, el nuevo panorama digital ha dado lugar a una transformación radical de la percepción que del periodismo manejan profesionales en activo, estudiantes o directivos de los medios. Como consecuencia, se ha producido un incremento sostenido de los estudios dedicados a analizar los fundamentos de la profesión en el nuevo entorno (Frith y Meech, 2007; MacDonald, 2006; Mensing, 2010), con aportaciones significativas en España (Diezhandino, 2012; Salaverría y Barrera, 2009; Pestano, Rodríguez Wangüemert y Del Ponti, 2011; Tuñez, 2010; Vivar, 2011).

Aunque Internet comienza a generalizarse en las redacciones durante el último lustro de los años noventa, a lo largo de esa misma década los estudios sobre la profesión siguieron insistiendo en una formación basada en ciertos "ítems sagrados", que aportaban escasas innovaciones más allá de la introducción de los nuevos soportes en un panorama de creciente convergencia: pulcras redacciones; selección y tratamiento escrupulosos de las fuentes informativas; y producción de noticias e historias en torno a ciertas rutinas y valores-noticia. Por otro lado, una parte importante de los estudios seguía dando preponderancia a la capacitación de profesionales para la industria y al fomento del trabajo experiencial en las redacciones, muchas veces con la figura de un mentor que ayudaba a completar las enseñanzas impartidas en el ámbito académico.

Pero la organización del newsroom ya es muy diferente a la del pasado, y comienza a representar el que puede ser considerado uno de los más grandes desafíos sufrido por el periodismo desde su aparición. Así, si antes de la irrupción tecnológica de los noventa, la profesión se hallaba un tanto "desmusculada", las mutaciones en el ecosistema informativo están y van a seguir afectando radicalmente a la relación entre periodistas y editores, y a las condiciones materiales en las que se produce el ejercicio profesional (Muro, 2012).

Andrés y Casero-Ripollés (2012: 130) resumen acertadamente estas mutaciones en dos procesos clave: el aumento del número de actores que intervienen en la cadena de producción de las noticias y cambios en el flujo informativo como: la aceleración en la difusión de noticias: el aumento de la flexibilidad en la distribución a través de múltiples plataformas; la abundancia comunicativa con la consiguiente fragmentación de la audiencia y la expansión global de la información. Estas últimas comportan, además, alteraciones profundas en las fórmulas tradicionales de búsqueda, producción y circulación de las noticias (Bivens, 2008), por lo que se suele afirmar que el entorno digital exige versatilidad a la vez que especialización. Por una parte, los perfiles polivalentes obligan al periodista a acumular labores antes ejercidas por especialistas de otras disciplinas - ej. documentación, fotografía, adaptación de contenidos a diferentes soportes, etc.- (Salaverría y García Avilés, 2008: 32). Por otra, se observa la irrupción de perfiles profesionales altamente especializados -periodistas de datos y de precisión, especialistas en narrativas digitales, gestores de comuni- 
dades virtuales, etc.- (Alguacil, 2012: 75-82), a la vez que desde el entorno laboral se demanda una creciente especialización técnica (Salaverría, 2012: 13) y temática (Micó, Masip y Ballano, 2012: 289).

En otro orden de cosas, el propio mercado de la información sigue sufriendo los vaivenes de la digitalización, que se evidencia en la confluencia de actores ajenos al campo de la información -industria informática, telecomunicaciones, agregadores de noticias, etc.-; una feroz competencia por el mercado publicitario; o una reconversión industrial, que, sumada a la crisis que atraviesa el sector, ha elevado la tasa de paro hasta cifras nunca alcanzadas.

Cabe destacar asimismo que frente al modelo dominante de formación periodística centrada en la industria (Mensing, 2010: 513), se observa asimismo una emergencia del periodismo de responsabilidad social, cívico o para el cambio social, con el objeto de proporcionar "al ciudadano la información que necesita para ser libre y capaz de gobernarse a sí mismo" (Kovach y Rosentiel, 2012: 18). De ahí que, parafraseando a Janowitz (1975), en el nuevo panorama digital coexisten cada vez más muestras de un periodismo movilizador (advocacy) frente al periodista tradicional gatekeeper, o mero filtrador de datos del entorno.

\section{Algunas pistas para abordar el futuro}

La investigación llevada a cabo entre un grupo muy representativo del actual panorama de medios en España -entre otros, televisiones generalistas (TVE, Telecinco y Antena 3); principales diarios online españoles, pertenecientes a grupos de comunicación (elmundo.es, elpais.es, elperiodico.es, lavanguardia.com, larazon.es, abc.es, 20minutos.es); y diarios nativos digitales con vocación generalista (elconfidencial.com, eldiario.es, lainformacion.com, infolibre.es, publico.es, lamarea.com, teinteresa.es, libertad digital, periodista digital, vozpopuli.com o zoomnews.es-, ha arrojado cierta luz a la hora de afrontar los retos de la digitalización en el ámbito formativo.

Al contrario de lo que pudiera pensarse hace pocos años, las respuestas a la pregunta sobre qué competencias y perfiles demandan estos medios, ofrecen respuestas poco unánimes. La búsqueda de periodistas polivalentes, y con un buen dominio de las herramientas digitales, tampoco es una constante, en consonancia con los resultados de estudios afines al nuestro (Micó, Masip y Ballano, 2012). Es decir, son más numerosas las incertidumbres e incluso interrogantes como: $¿$ Se han difuminado ya las fronteras entre los estudios de periodismo y los de comunicación audiovisual? ¿Debemos enseñar a nuestros estudiantes a producir noticias para diferentes soportes? ¿Hasta dónde debe alcanzar la especialización?

Por otra parte, existen discrepancias cuando los entrevistados ponderan las competencias profesionales de los estudiantes de periodismo, ya sea en prácticas de grado o de postgrado. Periodista Digital afirma, por ejemplo, que no se interesa por becarios 
o periodistas no aptos para realizar tareas habituales como montar una entrevista en vídeo $^{2}$. Por el contrario, el diario gratuito 20 Minutos, con edición impresa y digital y una amplia redacción, no encuentra dificultad en integrar y formar a los recién llegados y dispone incluso de un acuerdo con su comité de empresa para la incorporación anual de cierto número de becarios.

Ahora bien, el recurso a becarios o recién graduados con poca o nula experiencia ha sido, en los últimos tiempos, un recurso habitual de las todavía abultadas redacciones de los grandes diarios de referencia. Por su parte, los pure players buscan, casi siempre, perfiles mucho más experimentados. Tanto es así que la principal queja de muchos de sus directores es precisamente no disponer de profesionales capaces de conseguir y elaborar materiales de calidad. En otras palabras, no existe carencia alguna de periodistas con suficiente especialización tecnológica, sino más bien de redactores que reúnan también las destrezas que siempre han distinguido al "viejo periodismo";; esto es, la capacidad para generar noticias que distingan al medio.

En otro orden de cosas, una mayoría de profesionales entrevistados $-\mathrm{y}$ de acuerdo con una parte importante del colectivo académico- abogan por implementar la figura del "periodista multimedia", que, en términos un tanto polisémicos, vendría a ser sinónimo de un profesional polivalente o "anfibio", preparado para moverse "por tierra, mar o aire" (Gómez y Patiño, 2012), y capaz de desenvolverse al mismo tiempo en distintos medios, lenguajes y formatos (Martín Bernal, 2012). Ahora bien, esta polivalencia puede encubrir, en algunos casos, una reducción de plantillas, peores condiciones laborales y la consecuente pérdida de calidad y diversidad en la información. En este caso, la alternativa podría ser reforzar otra versión de la polivalencia que apostase por "rejuvenecer la mentalidad para explorar usos creativos en nuevas tecnologías" (Salaverría, 2012: 14), o por formar a periodistas que piensen "en clave multimedia" (Masip y Micó, 2008: 96).

Por otro lado, la mayoría de los medios entrevistados afirman que aún no se han llegado a emplear a fondo las grandes potencialidades que ofrece la red. Si bien Internet -como indica Salaverría (2010: 242)- suministra un entorno caracterizado por transformaciones como la hipertextualidad, la multimedialidad, la interactividad o la multitemporalidad, la mayor parte de los medios no han conseguido aún explotar las múltiples posibilidades de la convergencia ${ }^{4}$.

En relación con la mutación de las redacciones, destaca la composición de las plantillas de los nuevos medios, que disponen de un número reducido de efectivos: entre diez y veinte redactores, además de una serie de colaboradores con vinculaciones muy diversas. Sin embargo, todos los medios entrevistados son unánimes a la hora de considerar que solamente es posible elaborar una "información de calidad" con una redacción compuesta con no menos de cuarenta o cincuenta personas. Una cifra que solamente está al alcance de las redacciones integradas de los grandes diarios de referencia y de algunos medios regionales, mientras que en el caso de los diarios nativos digitales el único caso conocido es el del diario El Confidencial. 
Además, esta escasez de recursos humanos impide que se cubran todas las secciones habituales de la prensa tradicional, por lo que el resto de las informaciones y opiniones suelen proceder de agencias o son elaboradas por colaboradores con diferentes grados de experiencia y formación. En este sentido, el periodismo digital -tanto en las ediciones online de los grandes diarios como en los nuevos pure players- se enfrenta a los mismos problemas que han propiciado el claro declive de la prensa en la actualidad y entre los que podemos destacar la ausencia de redactores cualificados para generar noticias propias en ámbitos muy diversos, desde la política a la economía. No obstante, se detectan paralelamente tendencias innovadoras que pasan por cambios en la especialización temática tradicional de los diarios, y, por ende, en sus figuras periodísticas, como es el caso de JotDown.

Por último, cabe destacar que la universidad española lleva ya algún tiempo utilizando las TIC como palanca de cambio (Arribas, Choa y Sánchez Godoy, 2009). Hasta hace pocos años el foco de algunos docentes se centraba en los desarrollos tecnológicos (las herramientas utilizadas por el periodismo son vistas como universales, según Kunelius y Runsunoksa, 2008), que implicaban -por su elevado coste- una fuerte barrera de entrada para el acceso a la red de nuevos medios. Sin embargo, hoy día puede afirmarse que la tecnología ha devenido en una commodity; es decir, una mercancía sin un elevado coste monetario. De ahí que, desde La Razón y $A B C$ a eldiario.es o Informativos Telecinco.com empleen tecnología externalizada. En otras palabras, apenas existe desarrollo alguno de tecnologías propias particulares, puesto que existen grandes empresas proveedoras que operan exitosamente en este mercado. De hecho, un gasto excesivo en nuevos desarrollos tecnológicos solo permite hoy día una ventaja competitiva en el corto plazo, puesto que estos avances pueden ser "replicados" en muy poco tiempo y ofrecidos a un menor coste por las empresas tecnológicas que operan en el mercado.

\section{Conclusiones}

A la vista de los resultados, podemos entrever algunos desafíos evidentes para la docencia del periodismo. El primero de ellos es que estamos transitando hacia una nueva sociología del oficio del periodista. La selección del personal de las redacciones comporta, por mor de los reducidos presupuestos, equipos muy diferentes a las de los grandes diarios, y que se definen por su escaso número de efectivos, sus prolongadas jornadas laborales y los bajos salarios. Parece claro, como indicaba el periodista francés Bernard Poulet (2011: 258), que en este momento conviven, por una parte, una minoría de periodistas "vedettes", capaces de vender su firma, talento o "marca" por salarios más o menos elevados y, por otra, una masa muy amplia de periodistas anónimos y mal pagados. En medio, permanecería la "gruesa tropa" del periodismo tradicional, presente en todas las grandes redacciones, y que puede encontrarse en trance de extinción. Se percibe así que el papel de los reporteros y periodistas generalistas tiende progresivamente a disminuir, en consonancia con la opinión de John 
Tulloch (2011), quien cita a Malcom Gladwell, del New Yorker, para avalar su tesis de la decadencia del periodismo generalista: "journalism has to get smarter".

Del mismo modo, puede afirmarse que la red no ha supuesto una transformación radical del oficio periodístico tradicional; es decir, no ha supuesto -contra lo que se podía pensar- la expulsión de la profesión de una parte de los redactores "analógicos", o profesionales provenientes de la prensa papel, puesto que muchos de ellos han impulsado o liderado nuevos proyectos en la red, como es el caso de Público, El Diario, La Marea o Infolibre. La mayoría de estos periodistas no podrían recibir la apelación de "nativos digitales", ni suelen estar familiarizados con los usos y hábitos de la web. Sin embargo, su experiencia, su privilegiado acceso a las fuentes y la división del trabajo existente les permiten una dedicación completa a la búsqueda de noticias, con lo que depositan en las jóvenes incorporaciones su confianza en la utilización intensiva de recursos multimedia y la adopción de nuevas técnicas de storytelling.

Por otra parte, los nuevos entornos informacionales exigen que, sin renunciar a sus fundamentos, la universidad no deje de actualizar sus enseñanzas en "un panorama de transformaciones constantes con el fin de que los jóvenes graduados queden capacitados para desempeñarse profesionalmente en unos medios que reclaman nuevas destrezas" (Salaverría, 2010: 248). Esto es, conviene añadir a las habilidades habituales del "viejo periodismo" los nuevos conocimientos derivados de la propia red.

$\mathrm{Y}$ aunque es cierto que existe un evidente gap entre los periodistas tradicionales -muchos de ellos reciclados, no sin muchas dificultades, a Internet-, y los jóvenes procedentes de las facultades de comunicación, todavía es posible la coexistencia de ambos colectivos porque existe, al menos de momento, una evidente complementariedad entre ambos. Ahora bien, en el medio/largo plazo la tecnología ya no deberá ser patrimonio exclusivo de redactores muy avanzados, puesto que su desconocimiento -en opinión de bastantes medios- no permite usar todas las potencialidades de la red, y lo que es más grave, impide además diferenciar cualitativamente sus productos.

Una última consideración: aunque la carrera de periodismo siempre ha ejercido en todo el mundo un enorme atractivo entre muchos jóvenes, lo cierto es que, aún sin disponer de estadísticas fiables, solo una minoría de los recién egresados -por mor de la transformación de los modelos periodísticos- acaban trabajando en medios de comunicación en los que puedan ejercer su labor de periodistas. De ahí que sea posible que una insistencia constante sobre la conveniencia de priorizar el conocimiento y el uso de las nuevas herramientas tecnológicas y de otras habilidades propias de la profesión, pueda llegar incluso a desnaturalizar la esencia de lo que debe ser la formación universitaria: una enseñanza integral que ayude a los alumnos a analizar y descifrar procesos complejos y que les prepare para desarrollar su trabajo en entornos muy diversos, e incluso alejados de su objeto de estudio.

Por otro lado, además de incorporar nuevas asignaturas a los planes docentes de periodismo por exigencia del Espacio Europeo de Educación Superior (EEES), habría que reflexionar acerca del contenido que hoy ofrecen asignaturas enmarcadas 
con nuevas etiquetas como las de "ciberperiodismo", "periodismo online" o "periodismo en la red". Los planes de estudio de estas materias en España evidencian la insistencia en una formación exclusivamente técnica, en relación a la utilización de destrezas y nuevas herramientas que, en muchos casos, se tornan en obsoletas en unos pocos años. O lo que es lo mismo, debería tomarse en consideración si se debe seguir insistiendo o no en la capacitación en torno a las herramientas; o, por el contrario, primar una formación integral, y adaptada al nuevo ecosistema digital. Y si es así, habría que reconsiderar también como debería llevarse a cabo esta aproximación de carácter empírico.

\section{Bibliografía}

ALGUACIL SÁNCHEZ, S. M. (2012). "Ciberperiodismo: periodistas atrapados en la red”. En: TUÑEZ, M. (ed.). Viejo periodismo, nuevos periodistas. La Laguna: Sociedad Latina de Comunicación Social, p. 69-90.

ANDRÉS, R. y CASERO-RIPOLLÉS, A. (2012). Nuevas formas de producción de noticias en el entorno digital y cambios en el periodismo: el caso del 15-M. En: Comunicación y Hombre, $\mathrm{n}^{\circ}$ 8, p. 129-140.

APM (2012). Informe Anual de la Profesión Periodística. Madrid: APM.

ARRIBAS ALONSO, Y., CHOA MENDOZA, I. y SÁNCHEZ GODOY, T. (2009). Hacia la universidad digital 2010. Las TIC como palanca de cambio. En: Telos, $\mathrm{n}^{\mathrm{o}} 78$, p. 131-135.

BIVENS, R. K. (2008). "The internet, mobile phones, and blogging. How New media are transforming traditional journalism". En: Journalism Practice, vol. 2, n 1, p. 113-129.

DE AGUINAGA LÓPEZ, E. (2002). "El periodista en el umbral del siglo XXI". En: Estudios sobre el Mensaje Periodístico, no 8, p. 157-170.

DEUZE, Mark (2008). "What is multimedia journalism?” En: Journalism Studies, vol. $5, \mathrm{n}^{\circ} 2$, p. 139-152.

DIEZHANDINO NIETO, M. P. (2012). El periodista en la encrucijada. Barcelona: Ariel/Fundación Telefónica.

FRITH, S. y MEECH, P. (2007). "Becoming a journalist: Journalism education and journalism culture". En: Journalism, vol. 8, n 2, p. 137-164.

GÓMEZ Y PATIÑO, M. (2012). El cambio generacional: del periodista anfibio al comunicador de la era Amphibia”. En: TUÑ̃EZ LÓPEZ, M. (coord.): Viejo periodismo, nuevos periodistas. La Laguna: Sociedad Latina de Comunicación Social, p. 27-48.

JANOWITZ, M. (1975). "Professional models in journalism: the gatekeeper and the advocate". En: Journalism Quarterly, vol. 52, no 4, p. 618-662.

JOHNSTONE, J. W. C., SLAWSKI, E. J. y BOWMAN, W. W. (1976). The news people: a sociological portrait of American journalists and their work. Urbana: University of Illinois Press. 
KOVACH, B. y ROSENTIEL, T. (2012). Los elementos del periodismo. Todo lo que los periodistas deben saber y los ciudadanos esperar. Madrid: Aguilar.

KUNELIUS, R. y RUNSUNOKSA, L. (2008). "Mapping professional Imagination. On the potential of professional culture in the newspapers of the future". En: Journalism Studies, vol. 9, no 5, p. 662-678.

MARTÍN BERNAL, O. (2012). Retratos robot del periodismo en el entorno digital. Los nuevos perfiles profesionales y laborales: En: DIEZHANDINO NIETO, M. P. (coord.): El periodista en la encrucijada. Barcelona: Ariel/Fundación Telefónica, p. 79-111.

MACDONALD, I. (2006). "Teaching journalists to save the profession: a critical assessment of recent debates on the future of US and Canadian journalism education". En: Journalism Studies, no 7, p. 745-764.

MASIP MASIP, P. y MICÓ SANZ, J. L. (2008). El periodista polivalente en el marco de la convergencia empresarial. En: Quaderns del CAC, $\mathrm{n}^{\mathrm{o}} 31-32$, p. 83-89.

MENSING, D. (2010). "Rethinking (again) the future of Journalism Education". En: Journalism Studies, vol. 11, no 4, p. 511-523.

MICÓ SANZ, J. L., MASIP MASIP, P. y BALLANO MACÍAS, S. (2012). "Criterios de contratación y perfiles profesionales emergentes en los medios. Universidad y empresas informativas en época de crisis en Cataluña”. En: Ámbitos: Revista Internacional de Comunicación, ${ }^{\circ}$ 21, p. 281-294.

MIRANDO, J. A. (1995). "The First College Journalism Students: Answering Robert E. Lee's Offer of a Higher Education". En: Annual Meeting of the Association for Education in Journalism and Mass Communication, Washington D.C., 9-12 Agosto.

MURO BENAYAS, I. (2012). El cambio y los principios periodísticos. En: DIEZHANDINO NIETO, M. P. (coord.). El periodista en la encrucijada. Barcelona: Ariel/Fundación Telefónica, p. 25-43.

PESTANO RODRÍGUEZ, J. M., RODRÍGUEZ WANGÜEMERT, C. y DEL PONTI, P. (2011). Transformaciones en los modelos de formación de periodistas en España. El reto europeo. En: Estudios sobre el mensaje periodístico, $\mathrm{n}^{\mathrm{o}} 15$, $\mathrm{p}$. 95-118.

POULET, B. (2011). La fin des journaux et l'avenir de l'information. Paris: Gallimard.

PROJECT FOR EXCELLENCE IN JOURNALISM (2012). The State of the News Media. Disponible en: stateofthemedia.org. [29-10-2013].

PROJECT FOR EXCELLENCE IN JOURNALISM (2013). The State of the News Media. Disponible en: stateofthemedia.org. [29-10-2013].

REUTERS INSTITUTE FOR THE STUDY OF JOURNALISM (2012). Reuters Institute Digital News Report 2012: Tracking the Future of News. Oxford: University of Oxford. Disponible en: reutersinstitute.politics.ox.ac.uk. [29-10-2013].

SALAVERRÍA, R. (2010). ¿Ciberperiodismo sin periodistas? Diez ideas para la regeneración de los profesionales de los medios digitales. En: CAMPOS FREIRE, F. (coord.). El cambio mediático. Zarmora/Sevilla: Comunicación Social, p. 236-249. 
SALAVERRÍA, R. (2012). Medios y periodistas, ¿un futuro compartido?. En: Cuadernos Evoca, no 7, p. 11-14.

SALAVERRÍA, R. y BARRERA, C. (2009). "The Spanish journalism education landscape". En: TERZIS, G. (ed.). European Journalism Education. Chicago: Intelect. The University of Chicago Press, p. 319-330.

SALAVERRÍA, R. y GARCÍA AVILÉS, J. A. (2008). "La convergencia tecnológica en los medios de comunicación: retos para el periodismo". En: Tripodos, $\mathrm{n}^{\circ} 23$, p. 31-47.

SLOAN, W. D. (1990). Makers of the media mind: Journalism educators and their Ideas. Hillsdale, NJ: Lawrence Erlbaum.

STEPBENSON, H. (2011). "Foreword". En: TERZIS, G. (ed.): European Journalism Education. Chicago: Intelect. The Univesity of Chicago Press, p. 11-16.

TULLOCK, J. (2011). "We are all Dickheads now: Reflections on the future of journalism". En: MAIR, J. y LANCE KEEBLE, R. (Eds.). The Internet and Journalism Today. Face the Future: Tools for the Modern Media Age. Suffolk: Abramis.

TUÑEZ LÓPEZ, M. (coord.) (2010). Viejo periodismo, nuevos periodistas. La Laguna: Sociedad Latina de Comunicación Social

TÚÑEZ LÓPEZ, M., MARTÍNEZ SOLANA, Y. y ABEJÓN MENDOZA, P. (2010). "Nuevos entornos, nuevas demandas, nuevos periodistas". En: Estudios sobre el Mensaje Periodístico, $\mathrm{n}^{\circ}$ 16, p. 79-94.

VIZAR ZURITA, H. (2011). "TIC, Internet y el sector de la comunicación: nuevos perfiles profesionales". En: Telos, $\mathrm{n}^{\circ}$ 87, p. 58-62.

\section{Notas}

1 Esta investigación forma parte de las líneas de estudio del proyecto de investigación GDI-03 Comunicación de la Universidad Internacional de La Rioja (UNIR), titulado: "Modelos de negocio de la prensa en el ámbito digital".

2 A este respecto, desde Periodista Digital se afirma: "se pierde un tiempo precioso corrigiendo los errores de esas personas, y eso es algo que una pequeña redacción no se puede permitir".

3 En los momentos actuales parece claro que el intento de algunos grandes medios de despoblar las redacciones de experimentados periodistas y sustituirlos por recién licenciados o graduados de las Facultades de Periodismo ha solventado problemas puntuales de edición. Sin embargo, ello no ha permitido avanzar en la fidelización de las audiencias, el cobro por los contenidos, y mucho menos ha aportado dosis de calidad a los diarios.

4 A pesar de que la "convergencia" ya no suponga ningún problema en opinión unánime de todos los entrevistados. 


\section{Los autores}

José Vicente García Santamaría es profesor del Departamento de Periodismo y Comunicación Audiovisual de la Universidad Carlos III de Madrid e investigador principal del proyecto $\mathrm{i}+\mathrm{d}$ "Modelos de negocio de la prensa en el ámbito digital". Doctor en periodismo por la Universidad Rey Juan Carlos de Madrid, sus líneas de investigación habituales se orientan a la economía del audiovisual, la evolución de los grupos multimedia españoles, la gestión de derechos deportivos en televisión, y la comunicación de crisis.

Alejandro Barranquero Carretero es profesor del Departamento de Periodismo y Comunicación Audiovisual en la Universidad Carlos III de Madrid y miembro del Grupo de Investigación Medicación Dialéctica de la Comunicación Social (MDCS) en la Universidad Complutense de Madrid. Doctor en periodismo por la Universidad de Málaga, su investigación se centra en los campos de la comunicación para el desarrollo y el cambio social, periodismo ciudadano y ciberactivismo, y teoría crítica. 